\title{
Application of multi-objective optimization to structural damage estimation via model updating
}

\author{
Faisal Shabbir, Piotr Omenzetter ${ }^{*}$ \\ Department of Civil and Environmental Engineering, The University Of Auckland, Auckland, New \\ Zealand
}

\begin{abstract}
This paper presents a novel damage detection method which simultaneously updates the undamaged as well as damaged structure model in a multi-objective optimization (MOO) process. Structural health monitoring via analysis of modal data and model updating has received considerable attention in the previous decade. Such damage detection methods typically require an updated baseline model of the undamaged structure and the associated errors can become aggregated when this baseline model is subsequently used for damage detection. The use of multi-objective model updating alleviates those issues. A beam structure with and without damage has been used as an example and different noise levels have been added to the identified mode shapes. The results have been compared with single-objective model updating and it has been found that the proposed method is more efficient for accurate estimation of damage severity.
\end{abstract}

Keywords: multi-objective optimization, modal data, model updating, damage estimation.

\section{INTRODUCTION}

This paper presents a novel damage estimation method which simultaneously updates the damaged as well as undamaged structure model in a multi-objective optimization (MOO) process. The main aim of this paper is the application of a MOO technique to damage estimation problems to concurrently utilize the experimental data of both undamaged and damaged structure. Contemporary damage detection and estimation methods which are based on model updating typically require an updated baseline finite element (FE) model of the undamaged structure. The updated undamaged model is then compared with an updated damaged model for assessment of damage severity. There might be many errors associated with this model updating technique, e.g. experimental errors, updating procedure errors or parametric errors. These errors may be aggregated in the subsequent model updating runs.

In this paper, a damage estimation algorithm is being proposed which simultaneously updates undamaged as well as damaged structure model in the MOO process for improving performance of the damage estimation procedure. In order to evaluate the performance of the proposed approach, it is applied to a numerically simulated simply supported beam. Different noise levels are added to the identified mode shapes to assess the performance of the proposed procedure for accurate damage estimation in noisy conditions. A genetic algorithm (GA) has been used as the optimization tool in this paper as multi-objective GA is well implemented ${ }^{1}$. Compared to the single-objective optimization (SOO), which gives one optimal solution, multi-objective formulation gives a set of alternative solutions. A desirable solution can then be selected based on acceptable trade-off between the two objective functions (in this case related to the undamaged and damaged structure).

This paper is structured as follows: Section 2 describes the MOO. This is followed by model updating of a simulated beam using SOO and MOO under different noise levels in Section 3. The results of damage estimation using SOO and MOO are also detailed in Section 3 and conclusions drawn from the study are reported in Section 4.

\section{MULTI-OBJECTIVE OPTIMIZATION (MOO)}

An optimal solution for a physical system modeled using one objective function can be found using SOO. However, when two or more objective functions are used concurrently, the task of finding one or more optimal solutions is referred to as MOO. Many real world optimization problems involve multiple objectives. The extremist principle which

*p.omenzetter@auckland.ac.nz; phone 649 923-8138; fax 649 373-7462; www.auckland.ac.nz 
Return to your MySPIE To Do List at http://myspie.org and approve or disapprove this submission. Your manuscript will not be published without this approval. Please contact author_help@spie.org with any questions or concerns.

prioritizes one objective over the others may lead to erroneous results especially in cases where the rest of objectives are also important or interdependent. Selecting a solution which is optimal for only one objective may compromise the other objectives. Evolutionary algorithms are a popular approach to solve MOO problems using the concept of domination ${ }^{2}$. According to this concept, one solution dominates the other solution if the following two conditions are satisfied:

1. The first solution is not worse than the second solution in all objectives, and

2. The first solution is strictly better than the second solution in at least one objective.

Violation of any of the above conditions indicates that the first solution does not dominate the second solution. It is intuitive that if any solution dominates the other solution, then it is also better in the context of MOO. This concept of domination is used to find 'non-dominated' solutions. When all pair-wise comparisons have been made for a given finite set of solutions, we expect to have a set comprising a number of solutions which do not dominate one another. An important property of this set is that each of its solutions dominates all other solutions outside of this set. In other words, the solutions in this set are better compared to the rest of the solutions. This leads to the definition of Pareto optimality which states that among all solutions, a non-dominated set of solution are those which are not dominated by rest of the solutions. This concept of Pareto optimality leads to a set of solutions known as the Pareto optimal set. A rank is assigned to the $\operatorname{set}^{3}$. A plot of objective function values corresponding to Pareto optimal set gives the Pareto front. Two basic approaches have been mentioned in the literature to obtain the Pareto optimal set, namely, preference-based and evolutionary-algorithm-based MOO. While a large number of optimization runs is required to construct a Pareto front using the preference-based approach, only a single run is required using evolutionary-algorithm-based approach.

\subsection{Multi-objective evolutionary algorithm (MOEA)}

In multi-objective evolutionary algorithm (MOEA) based optimization, all the objectives are evaluated concurrently instead of converting them into a single-objective function. Evolutionary algorithms, such as GA, work concurrently on a population of genes and use genetic operators such as selection, crossover and mutation to obtain globally optimal solutions. This evolution mechanism helps to explore the trade-off between solutions with different blends and grades of objectives. Also, they do not require gradients of the objective function; their chance to reach global optimal solutions is increased. Detailed review of multi-objective techniques can be found in ${ }^{4}$.

Many variants of MOO, based on Pareto front approach and using multi-objective evolutionary algorithms (MOEAs), have been proposed ${ }^{4}$. These include NPGA (niched Pareto genetic algorithm), NSGA (non-dominated sorting genetic algorithm), MOMGA (multi-objective messy genetic algorithm), and SPEA (strength Pareto genetic algorithm). Nondominated sorted genetic algorithm-II (NSGA-II) is one of the most popular and efficient MOEAs and has been used in many studies in the last decade ${ }^{3,5-7}$. Therefore NSGA-II has been adopted for this study to investigate its effectiveness for damage detection and estimation via dynamic model updating. The general steps involved in NSGA-II are as follows:

1. Termination criteria based on the accuracy required and total number of generations is selected

2. A random population of chromosomes (solutions) is initialized

3. Values of objective functions for each of the chromosome are obtained

4. Different ranks are assigned to each of the solutions based on a non-dominated sorting algorithm to classify the population into fronts

5. Offsprings of the parent population are created by randomly arranging a duplicate copy of the parent solutions

6. A tournament selection of best solutions obtained from the previous step is performed

7. Cross over with assumed probability is performed on the parent solutions to form new offsprings

8. The new offsprings are mutated with a mutation probability

9. A non-dominated sorting is performed on the new offsprings and once again classifies all the solutions into fronts using a non-dominated sorting algorithm

10. If the termination criteria are achieved, stop, or else go to step 6.

After some trial and error, the following parameters of NSGA-II have been used in the present research:

Population size $=500$

Maximum number of generations $=200$

Stopping criterion (Tolerance of objective function value) $=1 \times 10^{-10}$

Cross over probability $=0.8$ 
Return to your MySPIE To Do List at http://myspie.org and approve or disapprove this submission. Your manuscript will not be published without this approval. Please contact author_help@spie.org with any questions or concerns.

Mutation probability $=0.2$ linearly decreases to 0 at the end of maximum generations

Pareto fraction (fraction of solutions to be kept in the first front) $=0.35$.

\section{APPLICATION TO DAMAGE ESTIMATION}

In the previous decade, numerous studies have been performed to establish the health of the structure under in-situ conditions $^{8,9}$. In the context of dynamic FE model updating, assessment of physical characteristics of the structure is done by comparing basic modal properties (such as natural frequencies and mode shapes) with their FE model counterparts.

A numerically simulated simply supported beam has been studied to demonstrate the effectiveness of the damage estimation method which simultaneously updates the undamaged as well as damaged structure model in a MOO process. The simulated beam has a total length of $5 \mathrm{~m}$ and was discretized into 10 elements as shown in Figure 1. The beam has a total depth of $0.2 \mathrm{~m}$ and width of $0.25 \mathrm{~m}$. The density of the beam was assumed as $2500 \mathrm{~kg} / \mathrm{m}^{3}$ and modulus of elasticity as $3.2 \times 10^{4} \mathrm{MPa}$. The area of the cross section was $0.05 \mathrm{~m}^{2}$ and moment of inertia was $1.66 \times 10^{-4} \mathrm{~m}^{4}$. A preliminary model of the beam was assumed as the one which has the aforementioned section properties and is referred to as the initial FE model (Figure 1a). In the simulated 'experimental' model for the undamaged structure, the moment of inertia (MOI) of all the elements of the initial FE model has been reduced by $10 \%$ as shown in Figure $1 \mathrm{~b}$. A $10 \%$ reduction was assumed in the undamaged model as initial FE models are usually developed based on design drawings and assumptions and may not accurately represent the undamaged in-situ structure. For the simulated 'experimental' damaged structure, MOI of element No. 5 has been further reduced from $10 \%$ to $60 \%$ (Figure 1c). This methodology is advantageous to check the effectiveness of the proposed approach in updating both undamaged and damaged models simultaneously. The modal analysis was carried out on the undamaged and damaged beam to obtain the first five natural frequencies and mode shapes. The values of the first five natural frequencies for the initial FE model, undamaged model and damaged model are shown in Table 1. It can be seen that the maximum difference between the frequencies from the initial FE model to undamaged beam is $5.41 \%$, and to the damaged beam it is $17.51 \%$. Only vertical degrees of freedoms are considered in this study.

INITIAL MODEL

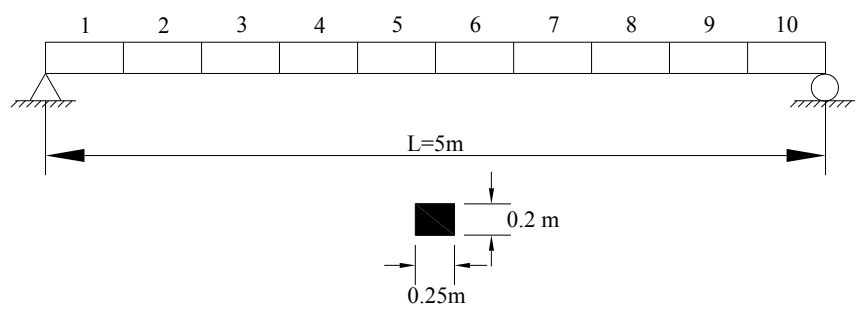

(a)

UNDAMAGED MODEL

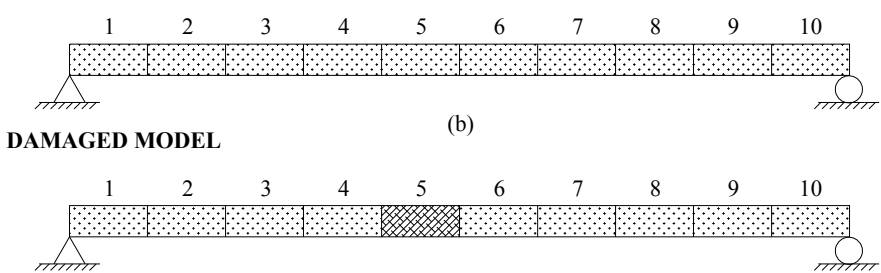

(c)

$$
10 \% \text { reduction in MOI } 60 \% \text { reduction in MOI }
$$

Figure 1. Simulated simply supported beam: (a) initial FE model without any reduction in MOI, (b) undamaged model with $10 \%$ reduction in MOI of all elements, and (c) damaged model with $60 \%$ reduction in MOI of element No. 5 and $10 \%$ in all other elements. 
Return to your MySPIE To Do List at http://myspie.org and approve or disapprove this submission. Your manuscript will not be published without this approval. Please contact author_help@spie.org with any questions or concerns.

Table 1. Frequencies of the simulated beam before model updating.

\begin{tabular}{|cccccc|}
\hline $\begin{array}{c}\text { Mode } \\
\text { No. }\end{array}$ & $\begin{array}{c}\text { Frequencies of } \\
\text { initial FE model } \\
\mathbf{( H z )}\end{array}$ & $\begin{array}{c}\text { Frequencies of } \\
\text { undamaged } \\
\text { beam (Hz) }\end{array}$ & $\begin{array}{c}\text { Frequencies of } \\
\text { damaged beam } \\
\mathbf{( H z )}\end{array}$ & $\begin{array}{c}\text { Difference } \\
\text { between initial } \\
\text { FE and } \\
\text { undamaged } \\
\text { model (\%) }\end{array}$ & $\begin{array}{c}\text { Difference } \\
\text { between initial } \\
\text { FE and damaged } \\
\text { model (\%) }\end{array}$ \\
\hline 1 & 12.98 & 12.31 & 11.04 & -5.41 & -17.51 \\
2 & 51.91 & 49.24 & 48.60 & -5.41 & -6.81 \\
3 & 116.73 & 110.74 & 103.13 & -5.41 & -13.19 \\
4 & 207.14 & 196.51 & 189.42 & -5.41 & -9.36 \\
5 & 322.11 & 305.58 & 291.86 & -5.41 & -10.37 \\
\hline
\end{tabular}

It can be assumed that modal frequencies are accurately determined in modal testing and experimental errors are usually present in the amplitude of mode shapes ${ }^{10}$. Consequently, some random noise has been added to each of the k-th component of the $\mathrm{j}$-th modal amplitude and the 'measured' component of the mode shape is given as:

$$
\Phi_{j k, \text { noise }}=\Phi_{j k}\left(1+\alpha_{\text {noise }} \varepsilon\right)
$$

where $\varepsilon$ is a random number between -1 and +1 and $\alpha_{\text {noise }}$ is the degree of noise. Three different noise levels were added in the mode shapes, i.e. $5 \%, 10 \%$ and $20 \%{ }^{11}$, for checking the effectiveness of the proposed approach. According to the usual procedure performed in actual tests, five data sets were considered for each noise level representing what would be repeated experiments. The updating parameter values reported later are the average of the five sets and their standard deviations.

A combined objective function related to the frequencies and model assurance criterion ${ }^{12}$ (MAC) is used in this study. The relative error between the experimental and analytical frequencies is:

$$
\Pi_{1}=\sum_{i=1}^{n}\left[\left(\omega_{a, i}-\omega_{e, i}\right) / \omega_{e, i}\right]^{2}
$$

where $\omega$ represents modal frequency, subscripts $a$ and $e$ refer to the analytical and experimental values, respectively, and $n$ is the total number of modes to be updated. The second objective function is related to the difference in mode shapes and can be defined in terms of MAC as:

$$
\Pi_{2}=\sum_{i=1}^{n}\left(1-\sqrt{M A C_{i}}\right)^{2} / M A C_{i}
$$

Two separate objective functions were defined for undamaged $\Pi_{\text {undam }}$ and damaged structure $\Pi_{\text {dam }}$ as follows:

$$
\begin{gathered}
\Pi_{\text {undam }}=\alpha \Pi_{1, \text { undam }}+\beta \Pi_{2, \text { undam }} \\
\Pi_{\text {dam }}=\alpha \Pi_{1, \text { dam }}+\beta \Pi_{2, \text { dam }}
\end{gathered}
$$

where weighting factors $\alpha$ and $\beta$ were taken as 1 .

Model updating of the undamaged and damaged beam has been performed in this section. The first five frequencies and mode shapes were selected to have a similar number of unknowns (MOI for each FE) as the number of knowns (frequencies and MACs). Parameter selection is an important step in model updating and prior damage detection and localization can result in an efficient selection of parameters in this study. Damage detection and localization has been the topic of interest in the previous decade and numerous techniques are available for detection and localization of damage $^{13-16}$. The damage detection and localization techniques are not the focus of this study and it was assumed that the location of damage is known to be in element No. 5 (Figure 1c). This information can effectively reduce the number of updating parameters in the damaged beam. Because of this, a total number of eleven parameters have to be updated in this case, which include all 10 element MOIs in the undamaged beam (Figure 1b) and one element (Element No.5) MOI 
Return to your MySPIE To Do List at http://myspie.org and approve or disapprove this submission. Your manuscript will not be published without this approval. Please contact author_help@spie.org with any questions or concerns.

in the damaged beam. Both $\mathrm{SOO}$ and $\mathrm{MOO}$ were performed to obtain the updated parameters to compare their performance.

Following the conventional approach, an updated undamaged model was obtained using Equation (4) and SOO. A total of 10 parameters were updated with 10 knowns, i.e. five frequencies and five MACs of the undamaged beam. After obtaining the updated undamaged model, the next step is to update the damaged model using Equation (5). As the damage location was assumed to be known, only element No. 5 was updated in this case and values of MOIs of the rest of the elements were taken from the updated undamaged model. One parameter was updated with 10 knowns in this case, i.e. five frequencies and five MACs of the damaged beam.

For MOO, a total of 11 parameters need to be concurrently updated, in which case 10 parameters belong to the undamaged beam and one parameter belongs to the damaged beam. Both Equation (4) and Equation (5) were concurrently used as two separate objective functions. The total number of knowns (frequencies and MACs) is 10 in this case and the number of unknowns (individual element MOIs) is 11 . The optimum solution is selected from the Pareto optimal set which minimizes the following expression:

$$
f=\sqrt{\Pi_{\text {undam }}^{2}+\Pi_{\text {dam }}^{2}}
$$

Figure 2a shows the mean errors in the stiffness ratio of the updated MOIs to their true values for $5 \%$ added noise for the undamaged and damaged beam for the five considered 'experiments', whereas Figure $2 b$ shows standard deviations of these ratios. The maximum mean error for single-objective updating is found to be 0.118 and the maximum mean error for multi-objective case is 0.056 . Likewise, the maximum standard deviation for the single-objective case is 0.156 and for the multi-objective case it is 0.138 . It can be seen from the errors in the updated stiffness ratios in Figure 2a that MOO has less error in the updated parameters than SOO, proving that the proposed approach is more efficient in updating both the undamaged and damaged model. It also shows that MOO is less sensitive to experimental errors than SOO. It should be noticed comparing the number of knowns vs. the number of unknowns that the multi-objective case considers 20 knowns against 11 unknowns, whereas the single-objective case considers 10 knowns against 10 unknowns. Thus more information is available to the optimization algorithm in the multi-objective case. The standard deviation of the updated stiffness ratios in Figure $2 \mathrm{~b}$ shows that the SOO generally has slightly more dispersion in the results than the MOO.

Similarly, Figure 4a and Figure 5a show the mean errors in the stiffness ratio of the updated MOIs to their true values for $10 \%$ and $20 \%$ added noise, respectively, for the undamaged and damaged beam for the five considered 'experiments', whereas Figure $4 \mathrm{~b}$ and Figure $5 \mathrm{~b}$ show standard deviations of these ratios. Similar observations can be made as noted earlier in the case of $5 \%$ noise level where MOO generally performs better than SOO. The maximum mean errors for the single-objective updating are found to be 0.062 and 0.229 , whereas the maximum mean errors for the multi-objective case are 0.076 and 0.139 for $10 \%$ and $20 \%$ noise, respectively. The maximum standard deviations for the singleobjective case are 0.183 and 0.211 , whereas for the multi-objective case they are 0.110 and 0.272 for $10 \%$ and $20 \%$ noise, respectively. Also, it has been noted that with the increase in noise level, the standard deviation of the stiffness ratios increases.

\section{8 - 46 V. 4 (p.5 of 10) / Color: No / Format: A4 / Date: 2012-03-12 05:41:39 PM}




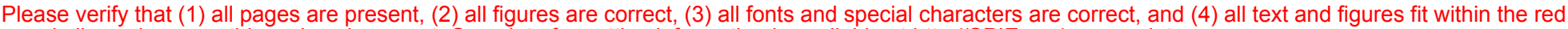
margin lines shown on this review document. Complete formatting information is available at http://SPIE.org/manuscripts

Return to your MySPIE To Do List at http://myspie.org and approve or disapprove this submission. Your manuscript will not be published without this approval. Please contact author_help@spie.org with any questions or concerns.

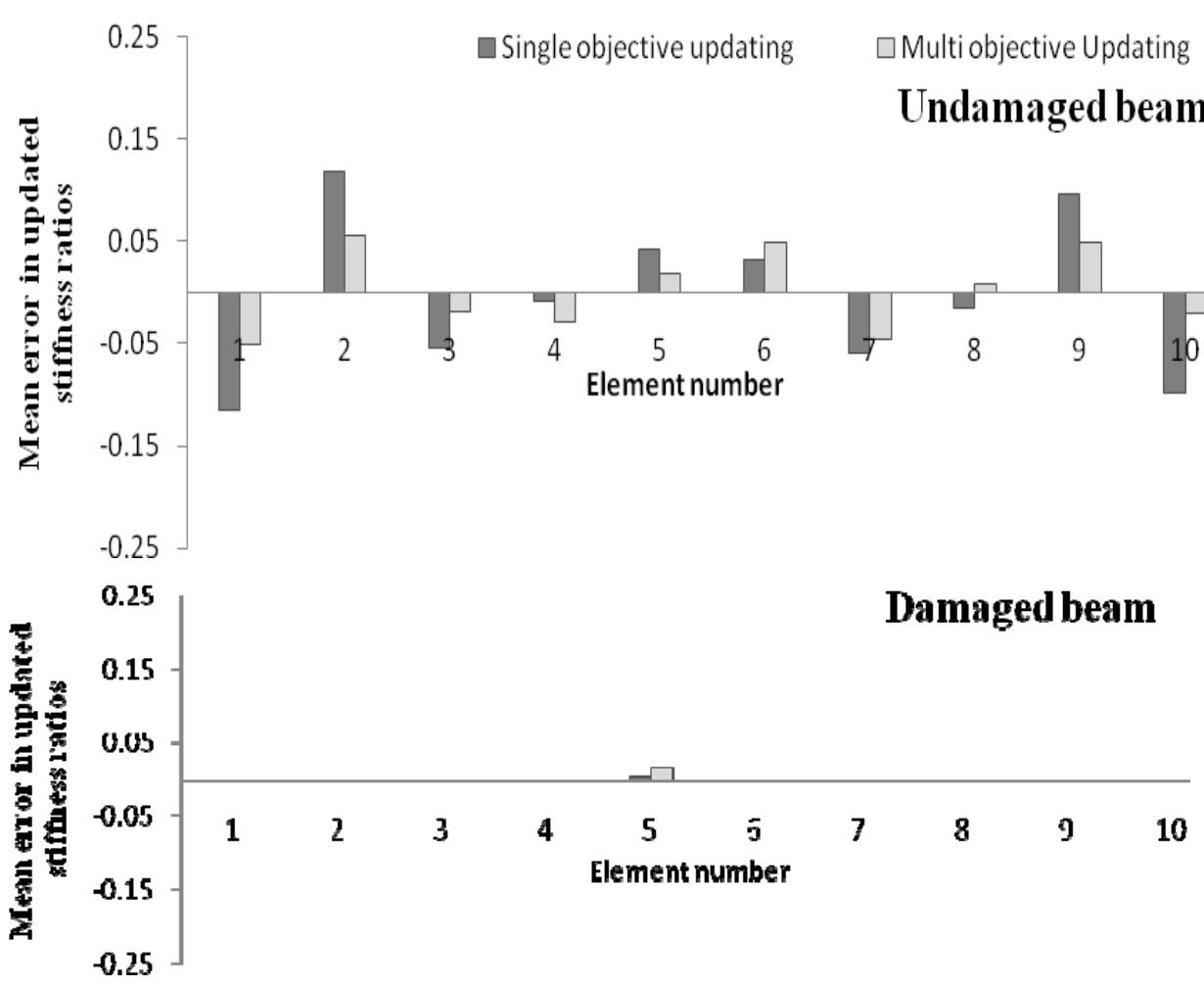

(a)
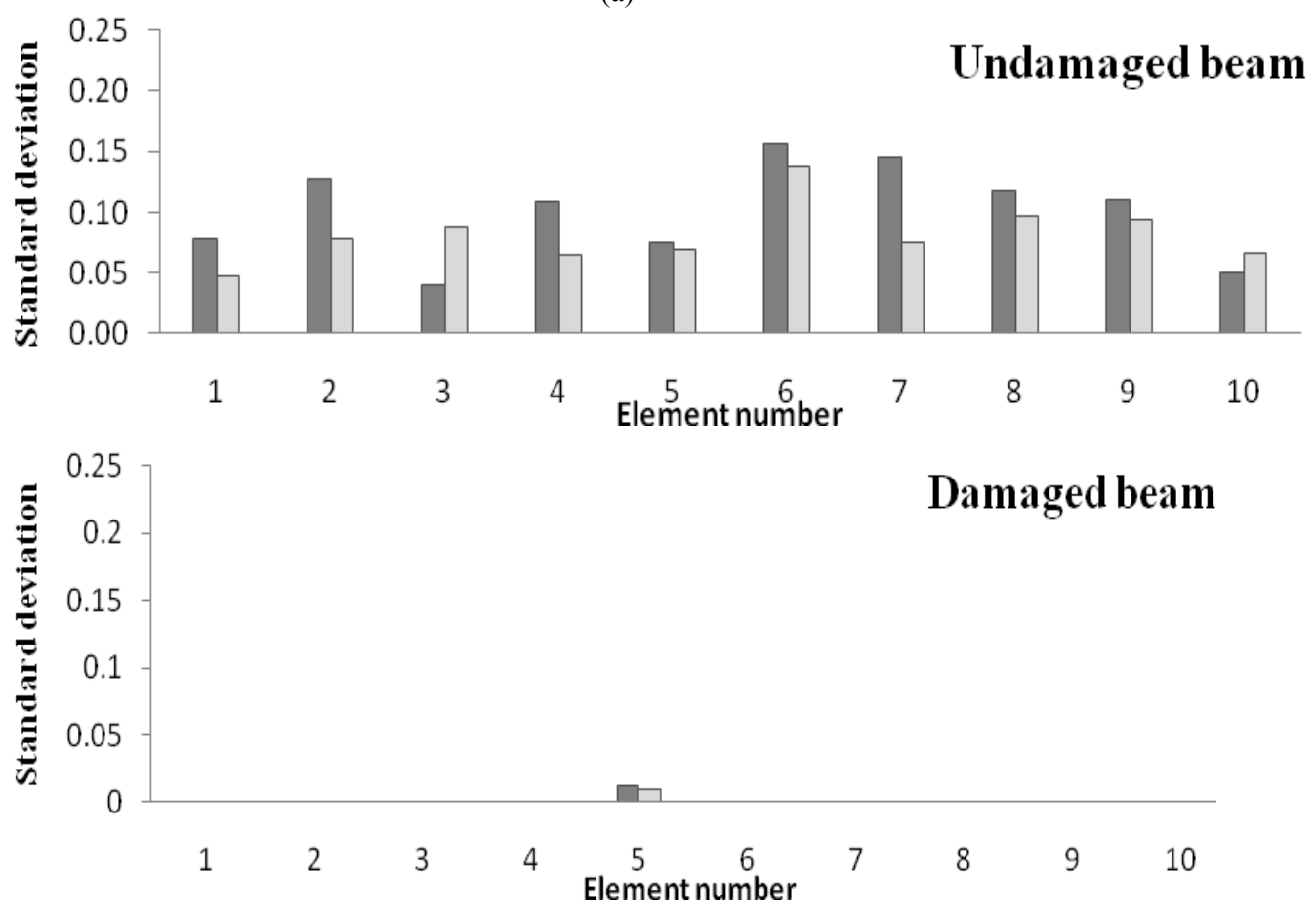

(b)

Figure 2. Damage estimation with 5\% noise level: (a) mean error in updated stiffness ratios, and (b) standard deviation of updated stiffness ratios. 
Return to your MySPIE To Do List at http://myspie.org and approve or disapprove this submission. Your manuscript will not be published without this approval. Please contact author_help@spie.org with any questions or concerns.
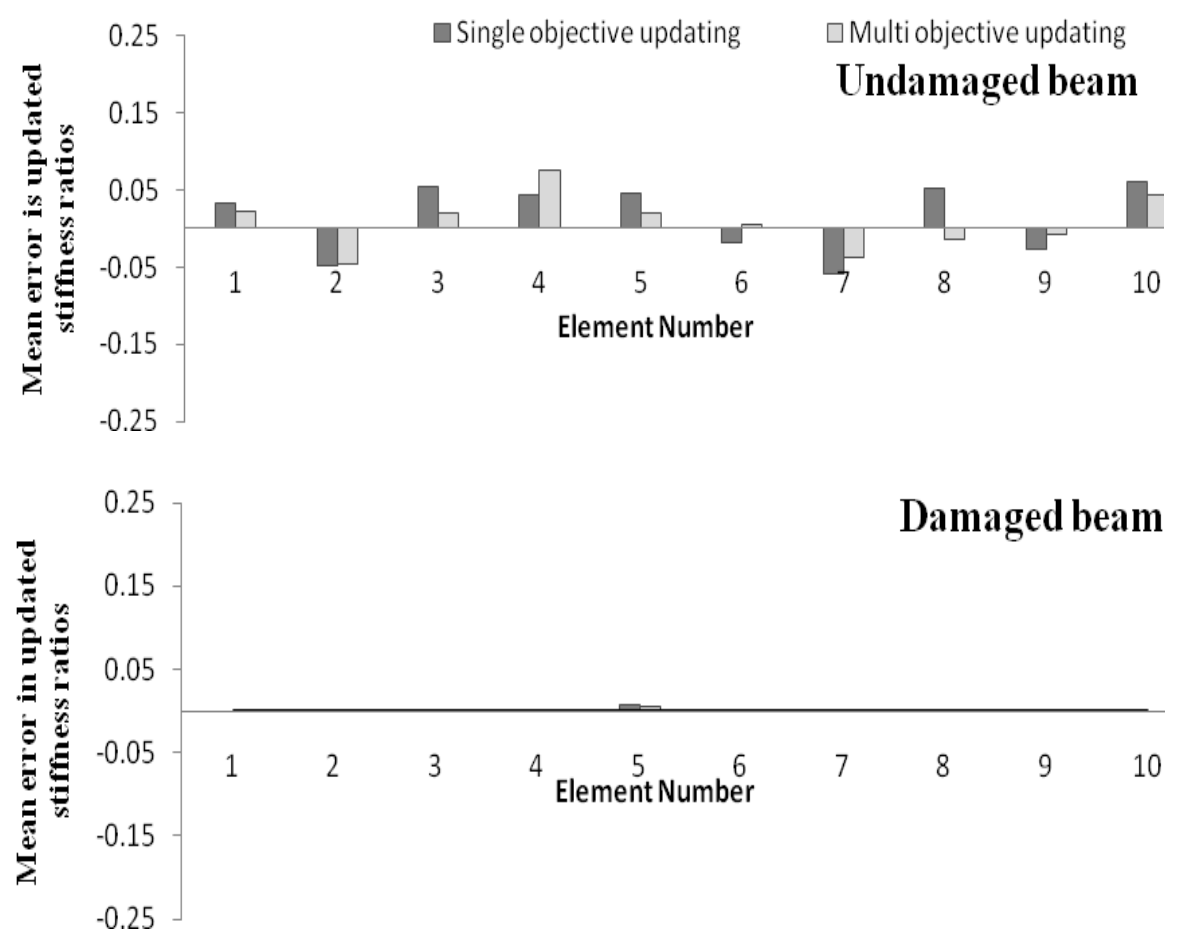

(a)
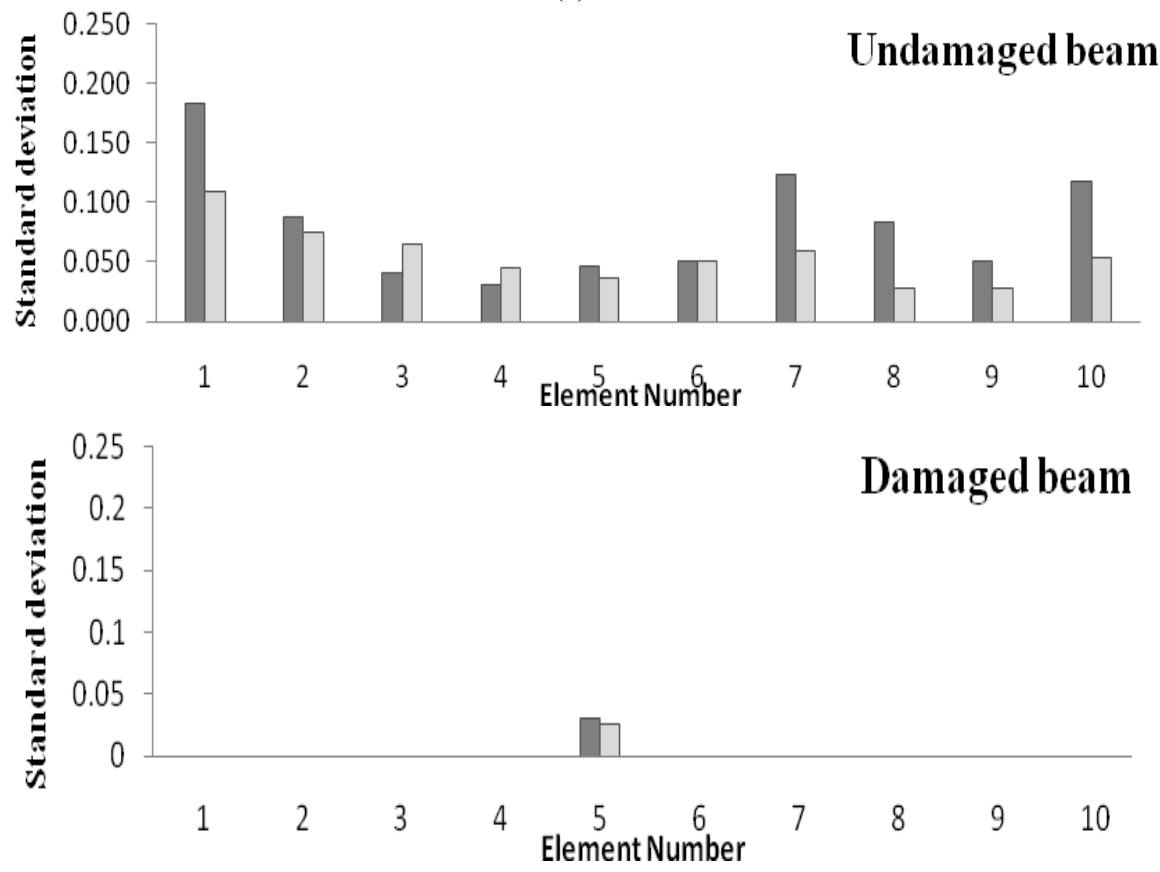

(b)

Figure 3. Damage estimation with 10\% noise level: (a) error in updated stiffness ratios, and (b) standard deviation of updated stiffness ratios. 
Return to your MySPIE To Do List at http://myspie.org and approve or disapprove this submission. Your manuscript will not be published without this approval. Please contact author_help@spie.org with any questions or concerns.
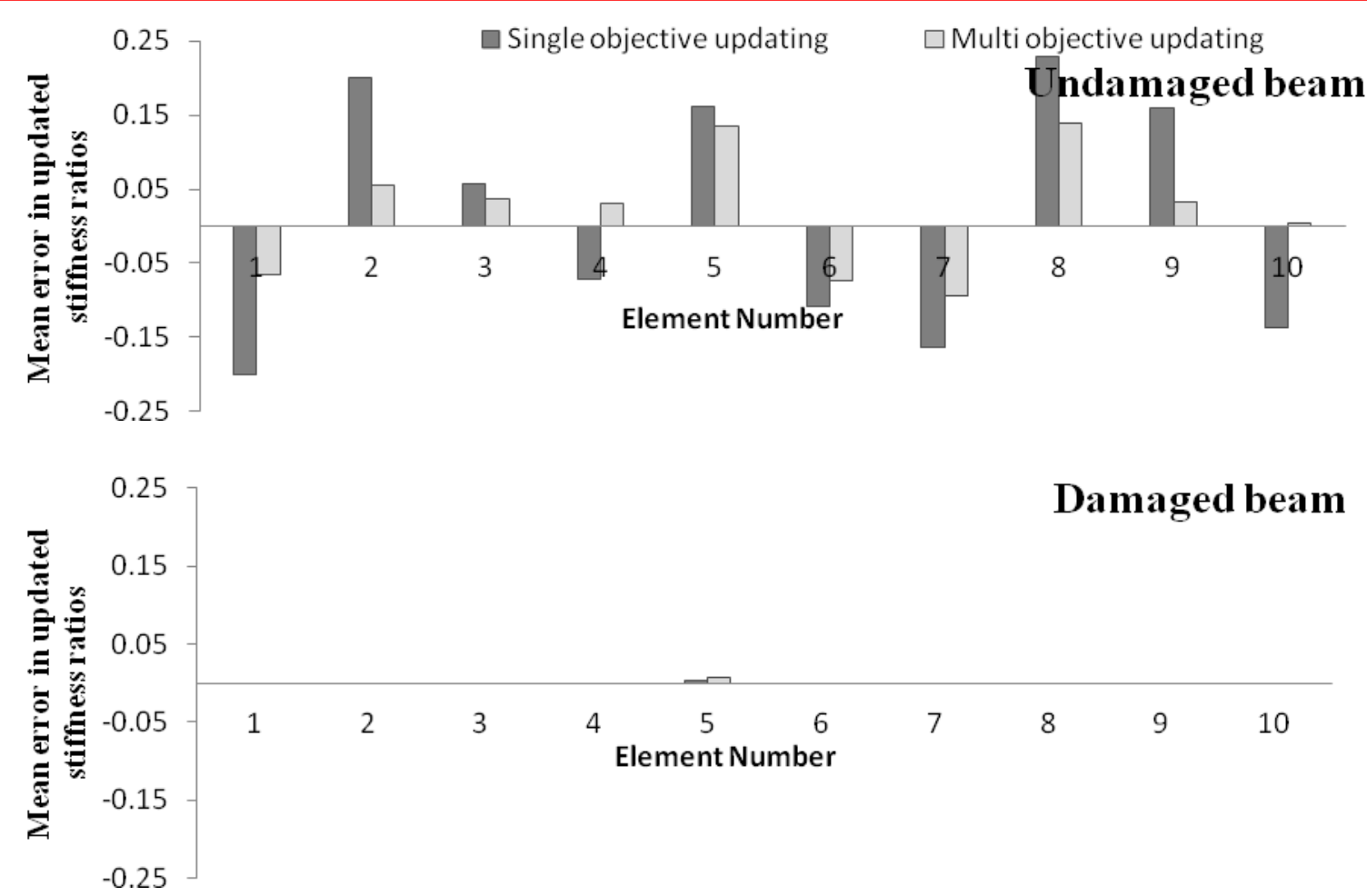

(a)
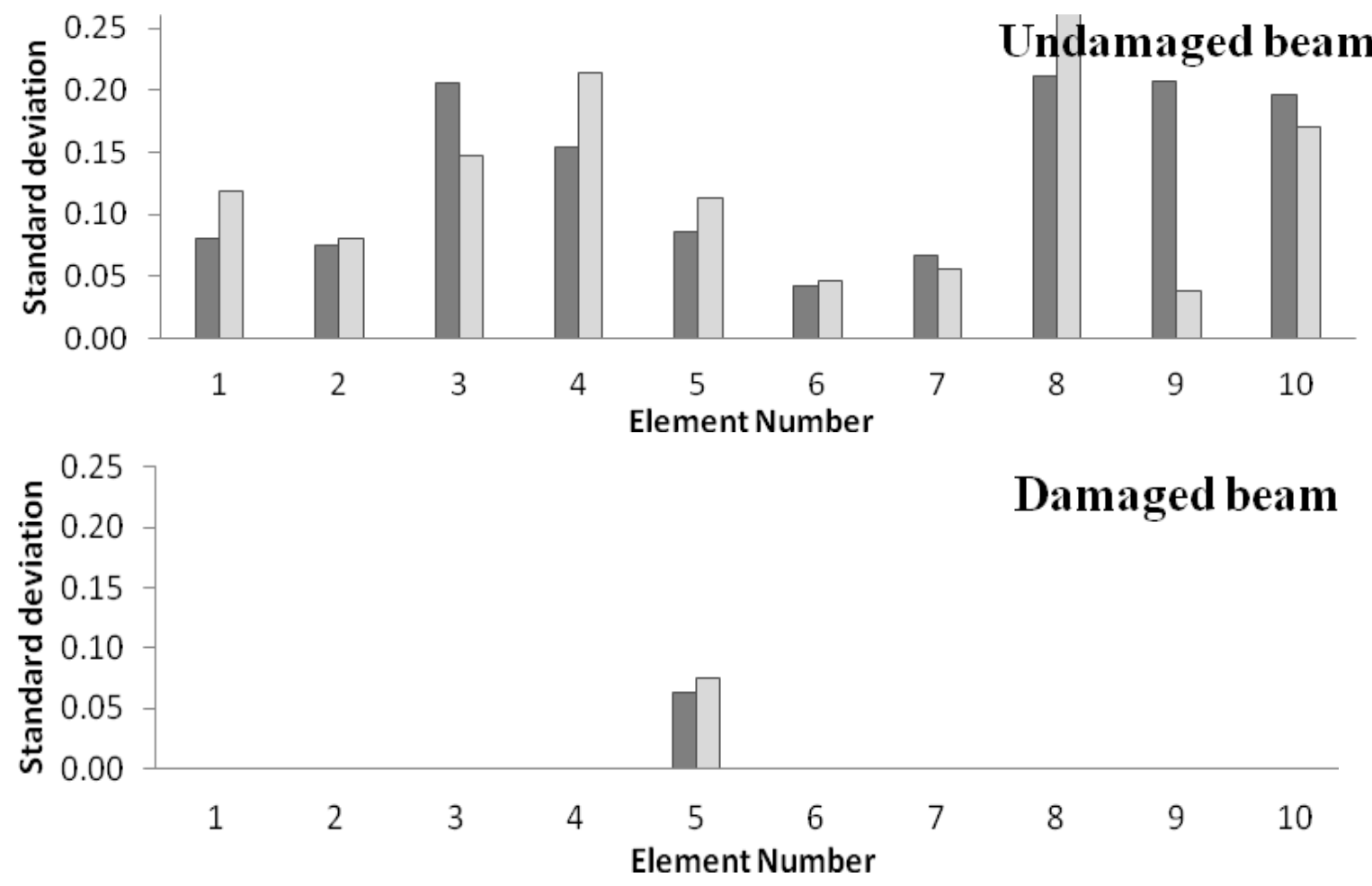

(b)

Figure 4. Damage estimation with 20\% noise level: (a) error in updated stiffness ratios, and (b) standard deviation of updated stiffness ratios. 
Return to your MySPIE To Do List at http://myspie.org and approve or disapprove this submission. Your manuscript will not be published without this approval. Please contact author_help@spie.org with any questions or concerns.

For assessment of damage severity, the updated undamaged model is compared with the updated damaged model for both SOO and MOO. Damage severity is estimated by subtracting the updated MOI ratios of the damaged model from that of the undamaged model. The actual damage index has been calculated as 0.5 for element No.5 in this study (as shown in Figure 1), which indicates a reduction of MOI of element No.5 from 0.9 to 0.4. The damage severity is estimated for element No.5 and shown in Figure 5 along with actual damage for all noise levels.

From the results, it can be seen that damage estimation from MOO is more accurate than from SOO. For example, the damage index for element No.5 (actual damage index $=0.5$ ) is found to be $0.540,0.538$ and 0.658 for the singleobjective case, whereas damage index for the same element is found to be $0.502,0.514$ and 0.628 for the multi-objective case for 5\%,10\% and 20\% noise, respectively. Generally speaking, SOO overestimates the damage severity to a higher degree than MOO. It can also be noticed that the increase in the noise level also affects the damage severity estimates, i.e. with the increase in noise levels, the damage was also over estimated to an even higher degree.

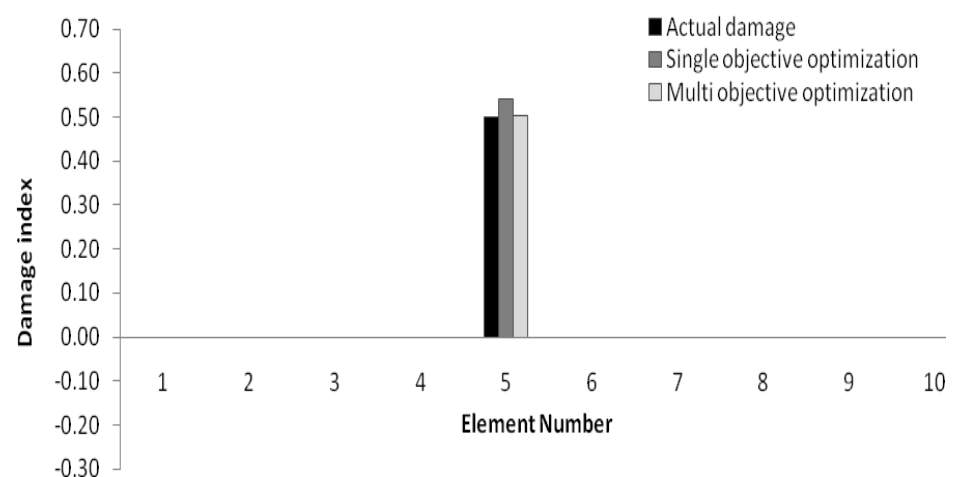

(a)

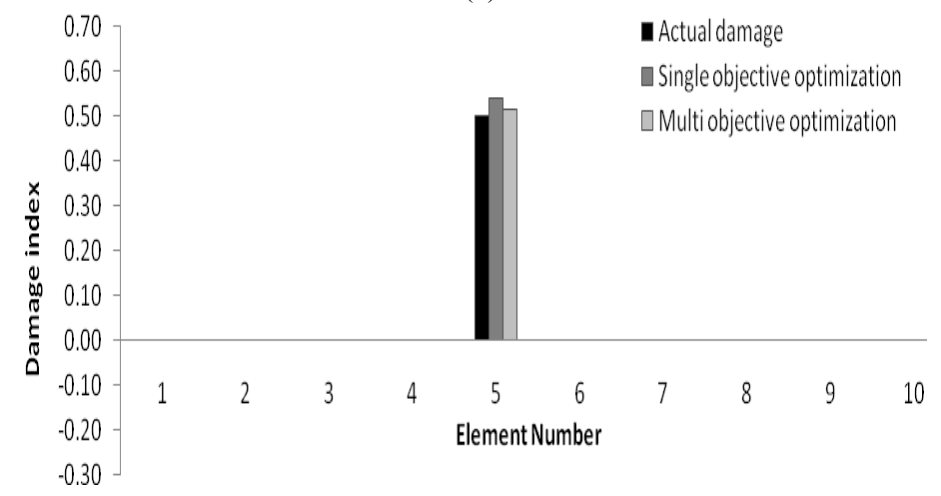

(b)

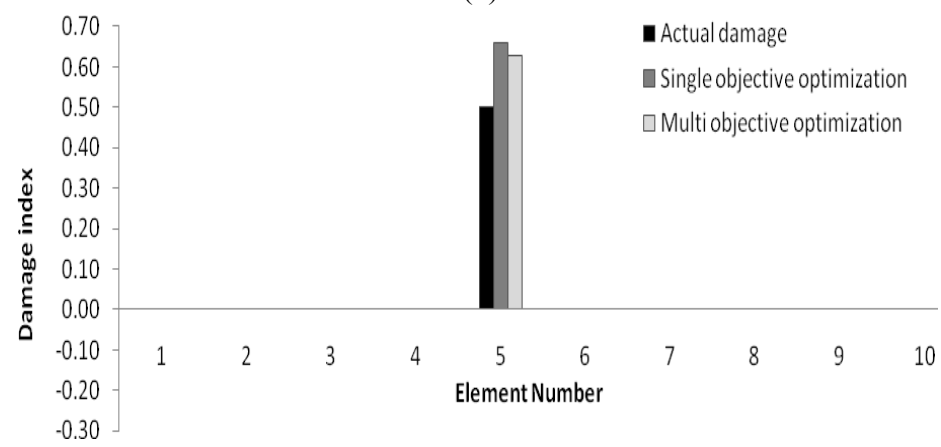

(c)

Figure 5. Damage severity estimation for element No.5 : (a) 5\% noise level, (b) $10 \%$ noise level, and (c) $20 \%$ noise level. 
Return to your MySPIE To Do List at http://myspie.org and approve or disapprove this submission. Your manuscript will not be published without this approval. Please contact author_help@spie.org with any questions or concerns.

\section{CONCLUSIONS}

This paper presents a damage estimation method which simultaneously updates the undamaged as well as damaged structure model in a MOO process. The following is the summary and conclusions of this study:

1. Damage estimation via structural modal updating typically requires an updated baseline model of the undamaged structure. The associated errors may propagate when this baseline model is subsequently used for damage estimation. The use of multi-objective updating alleviates these issues.

2. A numerically simulated simply supported beam has been used as an example problem and different noise levels of $5 \%, 10 \%$ and $20 \%$ have been added to the identified mode shapes to assess the performance of the proposed procedure in accurate estimation of the damage. It has been found that the proposed method has been successful in updating both undamaged and damaged models concurrently.

3. The results have been compared with the results obtained from single-objective model updating and it has been found that the proposed method is more efficient in accurately estimating damage severity and is less sensitive to experimental errors.

4. It has been concluded that with an increase in information of the damaged and undamaged system, better damage estimates were obtained.

\section{REFERENCES}

[1] Knowles, J., Corne, D. and Deb, K., Multiobjective problem solving from nature: From concepts to applications, Springer-Verlag, New York, (2008).

[2] Deb, K., Multi-objective optimization using evolutionary algorithms, John Wiley \& Sons Chichester, New York, (2001).

[3] Deb, K., Pratap, A., Agarwal, S. and Meyarivan, T., "A fast and elitist multiobjective genetic algorithm: NSGAII," IEEE Transactions on Evolutionary Computation 6 (2), 182-197 (2002).

[4] Coello, C. A. C. and Lamont, G. B., Applications of multi-objective evolutionary algorithms, World Scientific, NJ, (2004).

[5] Li, H. and Zhang, Q., "Multiobjective optimization problems with complicated Pareto sets, MOEA/D and NSGA-II," IEEE Transactions on Evolutionary Computation 13 (2), 284-302 (2009).

[6] Chan, R. R. and Sudhoff, S. D., "An evolutionary computing approach to robust design in the presence of uncertainties," IEEE Transactions on Evolutionary Computation 14 (6), 900-912 (2010).

[7] Koppen, M. and Yoshida, K., "Substitute distance assignments in NSGA-II for handling many-objective optimization problems," Lecture Notes in Computer Sciences 727-741 (2007).

[8] Begambre, O. and Laier, J. E., "A hybrid particle swarm optimization - simplex algorithm (PSOS) for structural damage identification," Advances in Engineering Software 40 (9), 883-891 (2009).

[9] Hu, N., Wang, X., Fukunaga, H., Yao, Z. H., Zhang, H. X. and Wu, Z. S., "Damage assessment of structures using modal test data," International Journal of Solids and Structures 38 (18), 3111-3126 (2001).

[10] Udwadia, F. E., "Structural identification and damage detection from noisy modal data," Journal of Aerospace Engineering 18 (3), 179-187 (2005).

[11] Perera, R. and Torres, R., "Structural damage detection via modal data with genetic algorithms," Journal of Structural Engineering, ASCE 132 (9), 1491-1501 (2006).

[12] Möller, P. W. and Friberg, O., "Updating large finite element models in structural dynamics," AIAA Journal 36 (10), 1861-1868 (1998).

[13] Yan, Y. J., Cheng, L., Wu, Z. Y. and Yam, L. H., "Development in vibration-based structural damage detection technique," Mechanical Systems and Signal Processing 21 (5), 2198-2211 (2007).

[14] Huh, Y. C., Chung, T. Y., Moon, S. J., Kil, H. G. and Kim, J. K., "Damage detection in beams using vibratory power estimated from the measured accelerations," Journal of Sound and Vibration 330 (15), 3645-3665 (2011).

[15] Chen, X., Ma, K., Wu, J. and Guo, X., "Damage detection through changes in frequency base on reinforced concrete beam," Advanced Materials Research 188-192 (2011).

[16] Xiang, J., Jiang, Z., Wang, Y. and Chen, X., "Study on damage detection software of beam-like structures," Structural Engineering and Mechanics 39 (1), 77-91 (2011). 